\title{
Immediate Improvement in Severe Mitral Regurgitation After Aortic Valve Replacement for Severe Aortic Insufficiency
}

\author{
Ahmad Zeeshan, MD ${ }^{1,2}$, Mojun Zhu, MD', John A. Elefteriades, MD ${ }^{1 *}$ \\ ${ }^{1}$ Aortic Institute at Yale-New Haven Hospital, Yale University School of Medicine, New Haven, Connecticut, USA \\ ${ }^{2}$ Heart and Vascular Institute, Cleveland Clinic, Cleveland, Ohio, USA
}

\begin{abstract}
A 57-year-old male with ascending aortic aneurysm, severe aortic regurgitation, and severe mitral regurgitation (MR) underwent ascending aortic replacement and aortic valve replacement. MR in this patient with normal mitral valve morphology was considered secondary to aortic valve incompetency. Consequently, a surgical approach to restore aortic valve function was adopted with successful MR resolution. This case report demonstrates the possibility of reversing early functional mitral regurgitation without surgically approaching the mitral valve. Copyright (c) 2016 Science International Corp.
\end{abstract}

\section{Key Words}

Functional mitral regurgitation - Aortic regurgitation - Aortic aneurysm

\section{Introduction}

Functional mitral regurgitation (FMR) is due to incomplete closure of mitral leaflets secondary to pathological left ventricular (LV) dilatation. In this case, aortic valve replacement (AVR) proved sufficient for resolving FMR secondary to aortic insufficiency in a patient without intrinsic anatomic mitral valve abnormalities.

\section{Case Presentation}

A 57-year-old male with history of wellcontrolled hypertension, smoking, and ascending

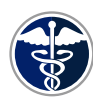

Fax +1 2037853552

E-Mail: aorta@scienceinternational.org

http://aorta.scienceinternational.org

\author{
(c) 2016 AORTA \\ Published by Science International Corp. \\ ISSN 2325-4637 \\ Accessible online at: \\ http://aorta.scienceinternational.org
}

aortic aneurysm was transferred from a community healthcare facility to the cardiac surgery intensive care unit of a tertiary care center after developing chest tightness, worsening dyspnea, orthopnea, and significantly decreased exercise tolerance. Acute myocardial infarction and aortic dissection were ruled out based on a normal electrocardiogram (ECG), normal levels of troponins, and a computed tomography angiogram (CTA) of the chest. The ascending aortic aneurysm was noted again. Approximately 4 months prior to this episode, a chest computed tomography for pneumonia had revealed this ascending aortic aneurysm incidentally. At the time of admission, his medications included furosemide $40 \mathrm{mg}$ four times daily and metoprolol $12.5 \mathrm{mg}$ twice daily. Physical examination revealed an afebrile, male with a body mass index of 28.7, heart rate of 78 per minute, respiratory rate of 20 per minute, blood pressure of 142/62 mm Hg, bibasilar rales, and 3+ diastolic and systolic heart murmurs, which were loudest at the base.

A preoperative transthoracic echocardiogram (TTE) revealed severe aortic regurgitation (AR); mild mitral, tricuspid, and pulmonic regurgitation; moderate left atrial (LA) dilatation, normal LV size with mild concentric hypertrophy, and normal systolic and diastolic function. The estimated ejection fraction was $64 \%$. Based on the TTE, the diameters of the visualized portions of the aorta were 4.0

* Corresponding Author:

John A. Elefteriades, MD

Aortic Institute at Yale-New Haven Hospital

Yale University School of Medicine

789 Howard Avenue, Clinic Building CB317, New Haven, CT 06519, USA

Tel.: +1 203785 2551; Fax: +1 203785 3552; E-Mail: john.elefteriades@yale.edu 
$\mathrm{cm}$ at the sinus of Valsalva, $3.4 \mathrm{~cm}$ at the sinotubular junction, $4.7 \mathrm{~cm}$ at the ascending aorta, and $3.7 \mathrm{~cm}$ at the aortic arch. Chest CTA with contrast showed $4.7-\mathrm{cm}$ dilatation of the mid-ascending aorta (Figure $1 \mathrm{~A}$ ) and atherosclerotic calcifications within the left anterior descending coronary artery (LAD). The aortic arch and descending aorta were of normal calibers. Cardiac catheterization exhibited single vessel coronary artery disease with $80 \%$ stenosis in the LAD and severe AR. The LV end diastolic pressure (LVEDP) was estimated to be 30-35 mm Hg.

Overall, this patient's symptoms were consistent with acute exacerbation of congestive heart failure secondary to aortic root dilatation and concomitant AR. With an aortic diameter greater than 4.5 $\mathrm{cm}$ and persistent chest tightness, he was at risk for aortic dissection, and a prompt repair of ascending aortic aneurysm was warranted. Intraoperative transesophageal echocardiogram (TEE) revealed moderate-to-severe mitral regurgitation (MR) with normal morphology of mitral leaflets in addition to previously noted severe $A R$ and ascending aortic aneurysm (Figure 2A). Both the anesthesia and surgical teams were very concerned about the severe $M R$, and we strongly considered approaching the valve surgically, in addition to the other necessary procedures. The patient underwent ascending aortic replacement, AVR, and coronary artery bypass grafting to the left anterior descending artery with left internal mammary artery. The mitral valve was not approached. Intraoperative TEE showed immediate improvement of $M R$ following the procedure (Figure 2B).

After surgery, the patient had an uneventful recovery and was discharged home on postoperative day 5. Two months later, chest CTA revealed normal caliber and contour of ascending aorta and aortic arch without any evidence of complication (Figure 1B).

\section{Discussion}

FMR is the most common etiology of MR in the United States [1]. Contrary to MR caused by diseases of valvular tissue such as flail leaflet and infective endocarditis, FMR is due to incomplete closure of mitral leaflets secondary to pathological LV dilatation. Chronic volume overload (as from AR) eventually overwhelms LV compliance and induces adverse ventricular remodeling, which gives rise to LV enlargement and dilatation of the mitral annulus. FMR develops when the mitral leaflets are no longer able to coapt. Chronic FMR may lead to further adverse remodeling of the heart including LA dilatation. When the compliance of LA exceeds a certain limit, LA pressure starts to rise, eventually resulting in pulmonary hypertension [2-7].

In this case, we surmised that restoring aortic valve competency (AVR) in the setting of normal systolic and diastolic functions and a morphologically normal mitral valve was likely to resolve FMR by returning left ventricular end diastolic volume (LVEDV) and LVEDP to normal levels. MR was immediately and
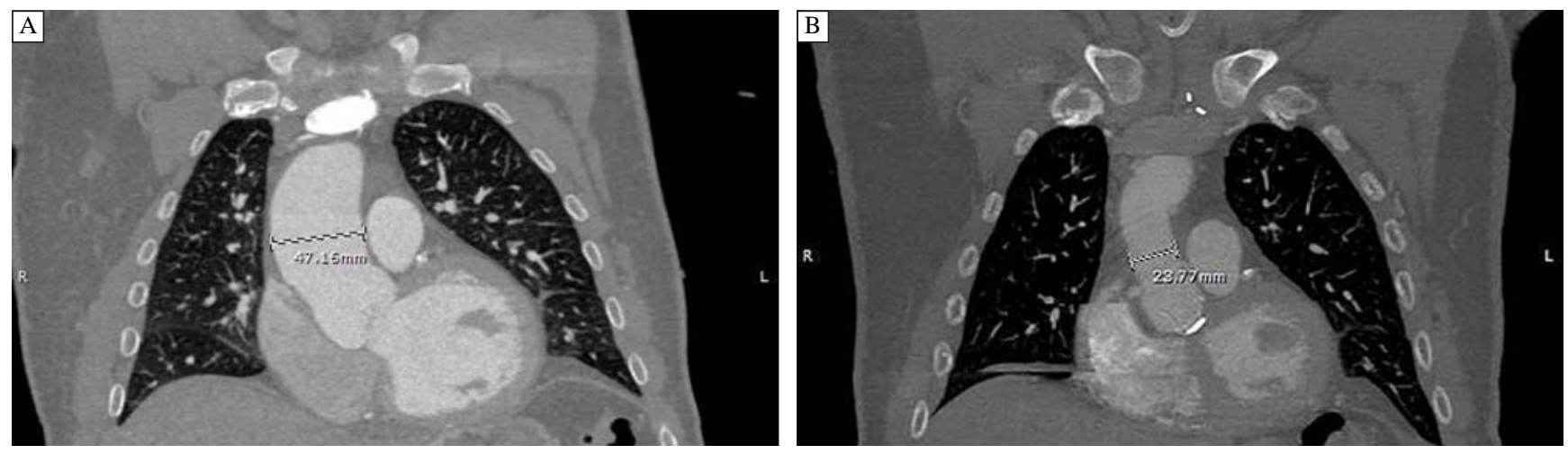

Figure 1. Panel A. Preoperative chest computed tomography angiogram showing an ascending aortic aneurysm with a 4.7-cm diameter. Panel B. Postoperative chest computed tomography angiogram showing an aorta with a normal caliber and contour. 

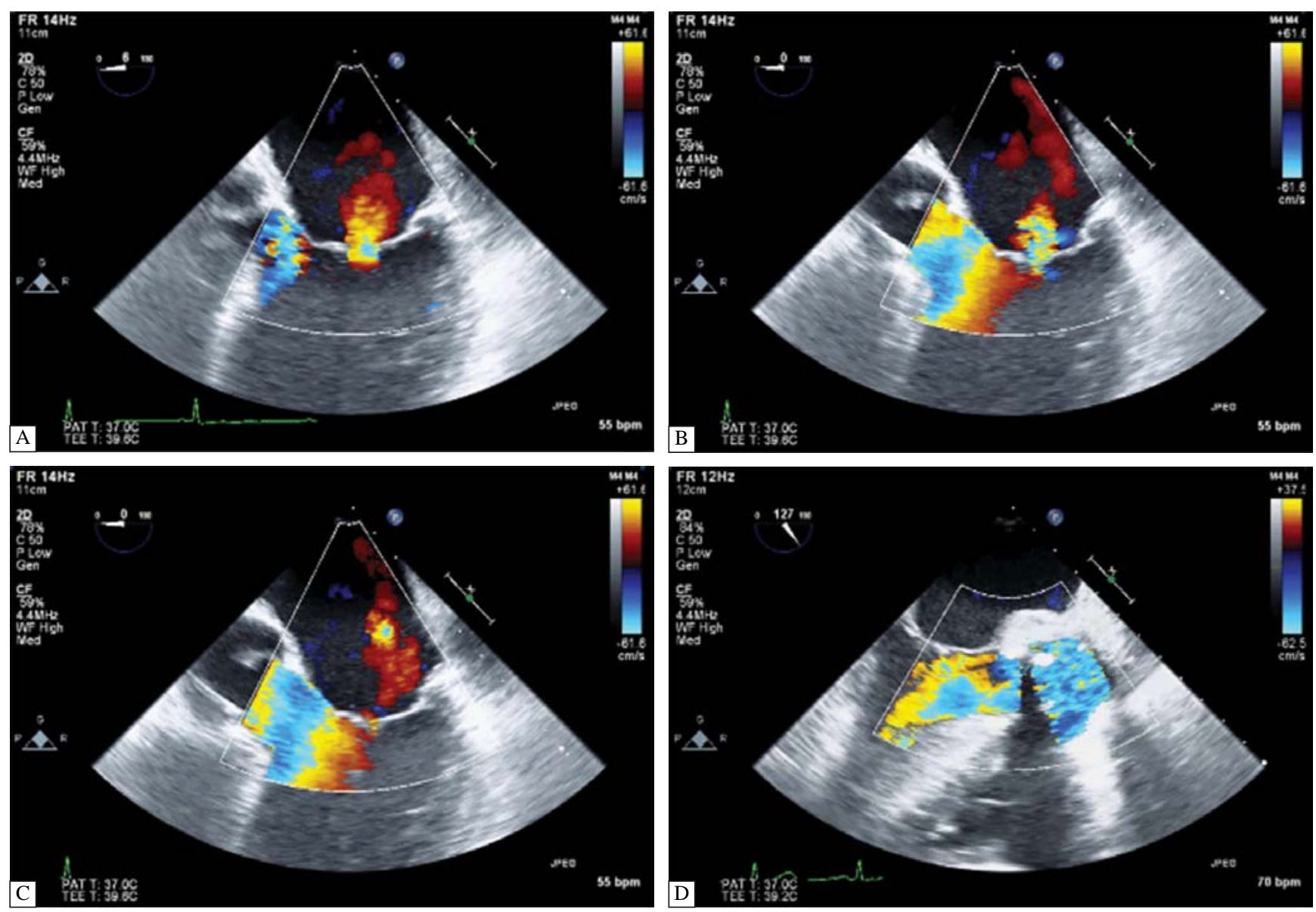

Figure 2. Panel $A, B$ and C: Preoperative transthoracic echocardiogram showing moderate to severe mitral regurgitation. Panel D: Postoperative transthoracic echocardiogram showing mitral competency.

dramatically improved in this patient following AVR. We were surprised and gratified to see immediate MR resolution upon elimination of the severe AR. In retrospect, our decision to avoid mitral valve surgery avoided unnecessary prolongation of an already major surgical procedure.

Double valve replacement has a reported mortality rate of $5.6-17.5 \%$, which is much higher than that of AVR alone (2.0-7.8\%) [6]. Thus, it is critical to evaluate the contribution of each individual lesion in order to choose the surgical treatment with the most favorable outcome. MR has long been considered a marker of impaired LV performance in aortic stenosis (AS) [8]. Mild-to-severe FMR in AS can be resolved with AVR alone, probably due to improved
LV function $[9,10]$. However, the efficacy and limitations of AVR for FMR secondary to AR have not been studied. Here, we report a successful reversal of functional MR after AVR. This case report highlights the relationship between valve function and ventricular geometry.

\section{Conflict of Interest}

The authors have no conflict of interest relevant to this publication.

Comment on this Article or Ask a Question 


\section{References}

1. de Marchena E, Badiye A, Robalino G, Junttila J, Atapattu S, Nakamura M, et al. Respective prevalence of the different carpentier classes of mitral regurgitation: a stepping stone for future therapeutic research and development. J Cardiac Surg. 2011;26:385-392. DOI: 10.1111/j.15408191.2011.01274.x

2. Glasson JR, Komeda M, Daughters GT, Bolger AF, Karlsson MO, Foppiano LE, et al. Early systolic mitral leaflet "loitering" during acute ischemic mitral regurgitation. J Thorac Cardiovasc Surg. 1998;116:193-205. DOI: 10.1016/S0022-5223(98)70117-5

3. Lai DT, Tibayan FA, Myrmel T, Timek TA, Dagum P, Daughters GT, et al. Mechanistic insights into posterior mitral leaflet inter-scallop malcoaptation during acute ischemic mitral regurgitation. Circulation. 2002;106:140-145. DOI: 10.1161/01. cir.0000032874.55215.82

4. Timek TA, Lai DT, Tibayan F, Liang D, Daughters GT, Dagum P, et al. Ischemia in three left ventricular regions: insights into the pathogenesis of acute ischemic mitral regurgitation. J Thorac Cardiovasc Surg. 2003;125:559-569. DOI:10.1067/mtc.2003.43

5. Nielsen SL, Hansen SB, Nielsen KO, Nygaard $\mathrm{H}$, Paulsen PK, Hasenkam JM. Imbalanced chordal force distribution causes acute ischemic mitral regurgitation: mechanistic insights from chordae tendineae force measurements in pigs. J Thorac Cardiovasc Surg. 2005;129:525-531. DOI: 10.1016/j. jtcvs.2004.07.044

6. Cohn LH. Cardiac surgery in the adult, 4th Edition. New York: McGraw-Hill Medical; 2013.

7. Di Mauro M, Gallina S, D'Amico MA, Izzicupo $P$, Lanuti $P$, Bascelli $A$, et al. Functional mitral regurgitation: from normal to pathological anatomy of mitral valve. Int J Cardiol. 2013;163:242-248. DOI: 10.1016/j. ijcard.2011.11.023

8. Schulman DS, Remetz MS, Elefteriades J, Frances CK. Mild mitral insufficiency is a marker of impaired left ventricular performance in aortic stenosis. J Am
Coll Cardiol. 1989;13:796-803. DOI: 10.1016/0735-1097(89)90218-0

9. Harris KM, Malenka DJ, Haney MF, Jayne JE, Hettleman B, Plehn JF, et al. Improvement in mitral regurgitation after aortic valve replacement. Am J Cardiol. 1997;80:741-745. DOI: 10.1016/S0002-9149(97)00506-7

10. Christenson JT, Jordan B, Bloch A, Schmuziger $M$. Should a regurgitant mitral valve be replaced simultaneously with a stenotic aortic valve? Tex Heart Inst J. 2000;27:350355. PMID: 11198307

Cite this article as: Zeeshan $A$, Zhu $M$, Elefteriades JA. Immediate Improvement in Severe Mitral Regurgitation After Aortic Valve Replacement for Severe Aortic Insufficiency. AORTA (Stamford) 2016;4(3):91-94. DOI: http://dx.doi. org/10.12945/j.aorta. 2016.15.035 